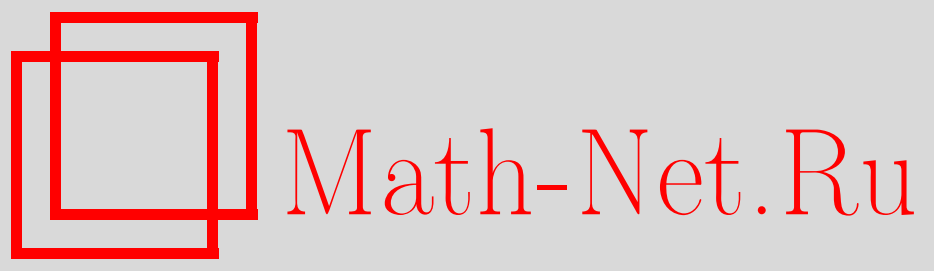

А. В. Домрина, Целочисленные характеристики решений некоммутативной сигма-модели, ТМФ, 2014, том 178, номер 3, 307-321

DOI: https://doi.org/10.4213/tmf8595

Использование Общероссийского математического портала Math-Net.Ru подразумевает, что вы прочитали и согласны с пользовательским соглашением http://www.mathnet.ru/rus/agreement

Параметры загрузки:

IP : 54.89 .56 .158

26 апреля 2023 г., 14:40:27

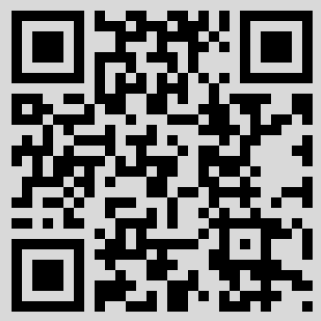




\title{
ЦЕЛОЧИСЛЕННЫЕ ХАРАКТЕРИСТИКИ РЕШЕНИЙ НЕКОММУТАТИВНОЙ СИГМА-МОДЕЛИ
}

\begin{abstract}
В некоммутативной сигма-модели у любого решения $\Phi$ конечной энергии имеются три неотрицательные целочисленные характеристики: нормированная энергия $e(\Phi)$, канонический ранг $r(\Phi)$ и минимальное унитонное число $u(\Phi)$. Доказаны неравенства $r(\Phi) \geqslant u(\Phi), e(\Phi) \geqslant u(\Phi)(u(\Phi)+1) / 2$. Также показано, что если числа $e, r, u \in \mathbb{N}$ удовлетворяют более сильным неравенствам $r \geqslant u$ и $e \geqslant r+u(u-1) / 2$, то существует решение $\Phi$ конечной энергии, для которого $e(\Phi)=e, r(\Phi)=r, u(\Phi)=u$.
\end{abstract}

Ключевые слова: некоммутативная сигма-модель, унитонная факторизация.

DOI: $10.4231 / \operatorname{tmf} 8595$

\section{1. ВВЕДЕНИЕ}

В классической унитарной сигма-модели теории поля изучаются гармонические отображения двумерной сферы в унитарную группу $U(n)$. Некоммутативная унитарная сигма-модель получается из классической посредством замены $U(n)$-значных функций на унитарные операторы в гильбертовом пространстве по правилам исчисления Вейля псевдодифференциальных операторов. Эта модель была предложена в связи с теорией струн в работе [1] и далее изучалась в работах [2]-[5]. В работе [4] был сформулирован ряд гипотез о строении пространств решений конечной энергии. В работе [5] по аналогии с коммутативным случаем [6], [7] было введено понятие петлевого поднятия и, в частности, доказано, что минимальное унитонное число решения конечной энергии равно степени его петлевого поднятия.

В настоящей работе мы продолжаем изучать свойства петлевого поднятия. Мы показываем, во-первых, что для натуральных чисел $e, u, r$, удовлетворяющих неравенствам $e \geqslant r+u(u-1) / 2, r \geqslant u$, существует решение, нормированная энергия которого равна $e$, канонический ранг равен $r$, а минимальное унитонное число равно $u$. Во-вторых, мы доказываем, что для любого решения $\Phi$ конечной энергии справедливы неравенства $e \geqslant u(u+1) / 2$ и $r \geqslant u$, где $e, u, r$ - соответственно нормированная энергия, минимальное унитонное число и канонический ранг решения $\Phi$.

${ }^{*}$ Московский государственный университет им. М. В. Ломоносова, Москва, Россия. E-mail: avdomrina@yandex.ru 
Отметим, что последние неравенства были сформулированы в работе [4] в виде гипотетического необходимого и достаточного условия существования решения с характеристиками $e, r, u$. Таким образом, мы устанавливаем их необходимость, а также достаточность несколько более сильных неравенств.

\section{2. ОБОЗНАЧЕНИЯ И ПРЕДВАРИТЕЛЬНЫЕ СВЕДЕНИЯ}

Пусть $H$ - сепарабельное гильбертово пространство со стандартным базисом $\left\{e_{0}, e_{1}, \ldots, e_{n}, \ldots,\right\}, a$ - оператор уничтожения, $a^{*}$ - оператор рождения,

$$
a e_{0}=0, \quad a e_{j}=\sqrt{j} e_{j-1}, \quad j=1,2, \ldots, \quad a^{*} e_{j}=\sqrt{j+1} e_{j+1}, \quad j=0,1, \ldots .
$$

Операторы $a, a^{*}$ отображают в себя векторное пространство

$$
D_{\infty}=\left\{\left.x \in H\left|\sum_{j=0}^{\infty}(j+1)^{k}\right| x_{j}\right|^{2}<\infty, k=0,1, \ldots\right\} .
$$

Пусть $M$ - множество унитарных операторов вида $\Phi=\mathrm{e}^{i \theta} I+K$, где $\theta \in \mathbb{R}$, $I$ - тождественный оператор, $K: H \rightarrow H$ - линейный оператор, образ которого $\operatorname{im} K=K(H)$ является конечномерным подпространством в $D_{\infty}$. Для каждого $\Phi \in M$ операторы

$$
\partial_{+} \Phi:=-\left[a^{*}, \Phi\right], \quad \partial_{-} \Phi:=[a, \Phi], \quad \Phi_{ \pm}:=\Phi^{-1} \partial_{ \pm} \Phi
$$

определены на пространстве $D_{\infty}$ и имеют конечномерный образ, лежащий в $D_{\infty}$. Поэтому эти операторы продолжаются по непрерывности на всё $H$. Они являются некоммутативными аналогами производных по $z, \bar{z}$ и соответствующих логарифмических производных. Наряду с $M$ мы также будем рассматривать подпространство $M_{0} \subset M$ унитарных операторов вида $\Phi=I+K$, где $I$ и $K-$ те же операторы, что и выше.

Некоммутативная $U(1)$-сигма-модель, которая изучалась в статьях [1]-[5], задается функционалом энергии

$$
4 e(\Phi)=\left\|\partial_{+} \Phi\right\|_{\mathrm{HS}}^{2}=\left\|\partial_{-} \Phi\right\|_{\mathrm{HS}}^{2}=\left\|\Phi_{+}\right\|_{\mathrm{HS}}^{2}=\left\|\Phi_{-}\right\|_{\mathrm{HS}}^{2}
$$

(здесь $\|X\|_{\mathrm{HS}}^{2}=\operatorname{tr} X X^{*}$ - квадрат нормы Гильберта-Шмидта оператора $X: H \rightarrow H$ с конечномерным образом).

Оператор $\Phi \in M$ называется решением некоммутативной $U(1)$-сигма-модели, если $t=0$ является критической точкой функции $e(\Psi(t))$ для любого гладкого отображения $\Psi:(-\epsilon, \epsilon) \rightarrow M$, удовлетворяющего условию $\Psi(0)=\Phi$. Отметим, что если $\Phi \in M$ - решение и $\theta \in \mathbb{R}$, то $\Psi=\mathrm{e}^{i \theta} \Phi$ - тоже решение. Такие решения называются эквивалентными. Тем самым любое решение $\Phi \in M$ эквивалентно некоторому решению $\Psi \in M_{0}$.

В работе [3] показано, что для любого решения $\Phi \in M$ число $e(\Phi)$ является неотрицательным и целым. При этом $e(\Phi)=0$ тогда и только тогда, когда $\Phi=\mathrm{e}^{i \theta} I$ для некоторого $\theta \in \mathbb{R}$. Число $e(\Phi)$ называется нормированной энергией решения $\Phi$. 
Пусть $\Phi \in M-$ произвольное решение. Ортопроектор $P: H \rightarrow H$ называется унитоном для $\Phi$, если $I-2 P \in M$ и справедливы равенства

$$
\begin{aligned}
P \Phi_{-} P^{\perp} & =0, \\
P^{\perp}\left(\Phi_{-}+2 a\right) P & =0 .
\end{aligned}
$$

Тогда (см. работу [3], утверждение 9) унитарный оператор $\Psi=\Phi(I-2 P)$ также является решением, причем

$$
\Psi_{-}=\Phi_{-}+2 \partial_{-} P .
$$

Операция добавления унитона обратима по формуле $\Phi=-\Psi\left(I-2 P^{\perp}\right)$, при этом $P^{\perp}$ является унитоном для решения $\Psi$. Каноническим рангом решения $\Phi$ называется число

$$
r(\Phi)=\operatorname{dimim} \Phi_{+}=\operatorname{dimim} \Phi_{-}=\operatorname{dimim} \partial_{-} \Phi=\operatorname{dimim} \partial_{+} \Phi .
$$

Согласно работе [3] (утверждения 11,12 ) для любого решения $\Phi \in M$ существует конечное число ортопроекторов $P_{0}, P_{1}, \ldots, P_{n}$ и число $\theta \in \mathbb{R}$ такие, что $P_{0}=0$ и

$$
\Phi=\mathrm{e}^{i \theta}\left(I-2 P_{0}\right)\left(I-2 P_{1}\right) \ldots\left(I-2 P_{n}\right),
$$

где $P_{j}-$ унитон для решения е $\mathrm{e}^{i \theta}\left(I-2 P_{0}\right)\left(I-2 P_{1}\right) \ldots\left(I-2 P_{j-1}\right)$ и $\operatorname{dimim} P_{j}<\infty$ для всех $j=1, \ldots, n$. При этом $e(\Phi)=\sum_{j=0}^{n} \operatorname{dim} \operatorname{im} P_{j}$. Разложение $(2.6)$ решения $\Phi$ в виде произведения конечного числа отражений вида $I-2 P_{j}$, где $P_{0}, P_{1}, \ldots, P_{j}$ удовлетворяют условиям, сформулированным выше, назовем унитонной факторизацией решения $\Phi$. Отметим, что представление (2.6) решения $\Phi$ не является единственным. Минимальным унитонным числом $и(\Phi)$ назовем наименьшее число $n \in\{0,1, \ldots\}$, для которого справедливо разложение $(2.6)$.

Пусть решение $\Phi \in M$ и ортопроекторы $P_{0}, P_{1} \ldots, P_{n}$ удовлетворяют соотношению (2.6). Операторнозначный многочлен

$$
\Phi(\lambda)=\prod_{j=0}^{n}\left(P_{j}^{\perp}+\lambda P_{j}\right)
$$

называется петлевым поднятием решения $\Phi$. В работе [5] показано, что петлевое поднятие решения $\Phi$ не зависит от выбора унитонной факторизации (2.6) этого решения и минимальное унитонное число решения $\Phi$ равно степени многочлена $\Phi(\lambda)$.

Рассмотрим более подробно решения $\Psi \in M$ с унитонным числом 0 или 1 . Согласно формулам (2.6) и (2.3) решение $\Psi$ эквивалентно отражению $\Phi=I-2 P$, где $V=\operatorname{im} P$ - конечномерное подпространство в $D_{\infty}$, удовлетворяющее условию $a(V) \subset V$. При этом $P=0$, если и только если $u(\Psi)=0$. Определенное выше отражение $\Phi$ называется $B P S$-решением ${ }^{1)}$, a $V=\operatorname{im} P$ называется $B P S$-подпространством. Строение BPS-решений описано в работе [4] (утверждение 1): для каждого BPS-решения $\Phi=I-2 P$ с соответствующим BPS-подпространством $V=\operatorname{im} P$ существует единственный комплекснозначный многочлен П $(z)$ со старшим членом, равным единице, такой, что $V=\operatorname{ker} \Pi(a)$. Верно и обратное: для каждого комплекснозначного многочлена $\Pi(z)$ со старшим членом, равным единице, множество

1) BPS-решения - решения Богомольного-Прасада-Зоммерфельда. 
$V=\operatorname{ker} \Pi(a)$ является BPS-подпространством. Для более детального описания BPSподпространств введем векторы

$$
R_{\lambda}=\sum_{j=0}^{\infty} \frac{\lambda^{j}}{\sqrt{j !}} e_{j}, \quad R_{\lambda}^{q}=\left(a^{*}-\bar{\lambda} I\right)^{q} R_{\lambda}, \quad \lambda \in \mathbb{C}, \quad q=0,1 \ldots
$$

Любой конечный набор векторов $R_{\lambda_{j}}^{q_{j}}$ линейно независим. Они удовлетворяют соотношениям (где угловые скобки означают линейную оболочку)

$$
\operatorname{ker}(a-\lambda I)=\left\langle R_{\lambda}\right\rangle, \quad \operatorname{ker}(a-\lambda I)^{k}=\left\langle R_{\lambda}, R_{\lambda}^{1}, \ldots, R_{\lambda}^{k-1}\right\rangle, \quad k \in \mathbb{N} .
$$

Разложим $\Pi(z)$ на множители: $\Pi(z)=\left(z-\lambda_{1}\right)^{k_{1}} \ldots\left(z-\lambda_{r}\right)^{k_{r}}$, где $\lambda_{i} \neq \lambda_{j}$ при $i \neq j$. Тогда справедливо представление

$$
V=\left\langle R_{\lambda_{j}}^{q_{j}} \mid 1 \leqslant j \leqslant r, 0 \leqslant q_{j} \leqslant k_{j}-1\right\rangle .
$$

При этом

$$
e(\Phi)=\operatorname{dim} V=k_{1}+\cdots+k_{r}=\operatorname{deg} \Pi, \quad r(\Phi)=r
$$

\section{3. ВСПОМОГАТЕЛЬНЫЕ УТВЕРЖДЕНИЯ}

УтВеРЖДЕНИЕ 1. Пусть $\Phi=\mathrm{e}^{i \theta} \prod_{j=0}^{n}\left(I-2 P_{j}\right)$ - унитонная факторизация решения $\Phi, \Phi_{l}=\prod_{j=0}^{l}\left(I-2 P_{j}\right), 1 \leqslant l \leqslant n$. Тогда

$$
\Phi_{l-}=2 \sum_{j=1}^{l}\left[a, P_{j}\right], \quad \Phi_{-}=2 \sum_{j=1}^{n}\left[a, P_{j}\right]
$$

ДОКАЗАТЕЛЬСТВо получается из (2.4) индукцией по $n$.

ЗАМЕЧАНИЕ 1. В условиях утверждения 1 соотношения (2.2) и (2.3) принимают вид

$$
\begin{aligned}
P_{k}\left[a, P_{1}+\cdots+P_{k-1}\right] P_{k}^{\perp} & =0, \quad 2 \leqslant k \leqslant n, \\
P_{k}^{\perp}\left(\left[a, P_{1}+\cdots+P_{k-1}\right]+a\right) P_{k} & =0, \quad 2 \leqslant k \leqslant n, \quad P_{1}^{\perp} a P_{1}=0 .
\end{aligned}
$$

УтВеРЖДЕНИЕ 2. Пусть $\Phi_{j}=I-2 P_{j}, j=1,2,-$ два ВPS-решения такие, что подпространства $V_{j}=\operatorname{im} P_{j}$ удовлетворяют включениям $V_{2} \subset V_{1}, a^{*} V_{2} \subset V_{1}$. Тогда $r\left(\Phi_{1}\right) \geqslant r\left(\Phi_{2}\right) u \operatorname{dim} V_{1} \geqslant r\left(\Phi_{1}\right)+\operatorname{dim} V_{2}$.

ДокАЗАТЕЛЬСтво. Пусть $\Pi_{j}(z)$ - многочлен со старшим коэффициентом, равным единице, такой, что $V_{j}=\operatorname{ker} \Pi_{j}(a), j=1,2$. Разложим $\Pi_{2}(z)$ на множители:

$$
\Pi_{2}(z)=\prod_{j=1}^{r\left(\Phi_{2}\right)}\left(z-\lambda_{j}\right)^{k_{j}}
$$

Согласно формулам (2.7)-(2.9)

$$
V_{2}=\left\langle R_{\lambda_{j}}^{q_{j}} \mid 1 \leqslant j \leqslant r\left(\Phi_{2}\right), 0 \leqslant q_{j} \leqslant k_{j}-1\right\rangle .
$$


Справедливо равенство $a^{*} R_{\lambda_{j}}^{q_{j}}=R_{\lambda_{j}}^{q_{j}+1}+\bar{\lambda}_{j} R_{\lambda_{j}}^{q_{j}}$, и по условию $V_{2}+a^{*} V_{2} \subset V_{1}$, отсюда $R_{\lambda_{j}}^{k_{j}} \subset V_{1}$, следовательно, BPS-подпространство

$$
\left\langle R_{\lambda_{j}}^{q_{j}} \mid 1 \leqslant j \leqslant r\left(\Phi_{2}\right), 0 \leqslant q_{j} \leqslant k_{j}\right\rangle \subset V_{1} .
$$

Поэтому многочлен $\Pi_{1}(z)$ делится на произведение $\Pi_{2}(z) \prod_{j=1}^{r\left(\Phi_{2}\right)}\left(z-\lambda_{j}\right)$. Таким образом, число различных корней многочлена $\Pi_{1}$ не меньше числа различных корней многочлена $\Pi_{2}$, т. е. $r\left(\Phi_{1}\right) \geqslant r\left(\Phi_{2}\right)$ и $\operatorname{deg} \Pi_{1} \geqslant r\left(\Phi_{1}\right)+\operatorname{deg} \Pi_{2}$. Остается учесть, что $\operatorname{deg} \Pi_{j}=\operatorname{dim} V_{j}, j=1,2$. Утверждение доказано.

\section{4. ПРИМЕР, ОБОБЩАЮЩИЙ ДИАГОНАЛЬНЫЕ РЕШЕНИЯ}

В следующей теореме строится класс решений, содержащий диагональные решения, которые изучались в работах [3], [5].

Tеорема 1. Пусть $\alpha_{1} \supset \cdots \supset \alpha_{n}-B P S$-подпространства такие, что $\alpha_{n} \neq\{0\}$ и $a^{*} \alpha_{j+1} \subset \alpha_{j}$ при $1 \leqslant j \leqslant n-1$. Рассмотрим ортопроекторы $P_{j}, 1 \leqslant j \leqslant n$, для которых $\operatorname{im} P_{j}=\alpha_{j}$. Положим $r_{j}=r\left(I-2 P_{j}\right)$. Тогда оператор $\Phi=\prod_{j=1}^{n}\left(I-2 P_{j}\right)$ является решением, удовлетворяющим соотношениям

$$
u(\Phi)=n, \quad e(\Phi)=\sum_{j=1}^{n} \operatorname{dim} \alpha_{j}, \quad r(\Phi)=\sum_{j=1}^{n} r_{j} .
$$

При этом

$$
r_{1} \geqslant r_{2} \geqslant \cdots \geqslant r_{n}, \quad e(\Phi) \geqslant \frac{n(n-1)}{2}+r(\Phi)
$$

ДокАЗАТЕЛЬСТво. Положим $\Phi_{0}=I, \Phi_{j}=\prod_{k=1}^{j}\left(I-2 P_{k}\right)$ для $1 \leqslant j \leqslant n$. Индукцией по $j$ убедимся, что $\Phi_{j}$ - решение, причем $P_{j}-$ унитон для $\Phi_{j-1}$.

Поскольку $\alpha_{1}$ - BPS-подпространство, $\Phi_{1}=I-2 P_{1}-$ BPS-решение и $P_{1}-$ унитон для $\Phi_{0}$, следовательно, при $j=1$ утверждение справедливо.

Предположим, что утверждение индукции справедливо при $j<k \leqslant n$. Покажем, что оно верно и при $j=k$. Достаточно проверить, что $P_{k}-$ унитон для $\Phi_{k-1}$, т. е. ортопроекторы $P_{1}, \ldots, P_{k}$ удовлетворяют соотношениям (3.1) и (3.2). Покажем, что

$$
\left[a^{*}, \sum_{l=1}^{k-1} P_{l}\right] P_{k}=0
$$

и тем самым выполнено равенство (3.1). Действительно, $\alpha_{l}$ - BPS-подпространство, следовательно, $P_{l} a^{*} P_{l}^{\perp}=0$ и $\left[a^{*}, P_{l}\right]=P_{l}^{\perp} a^{*} P_{l}$. При $l<k$ имеют место включения $\alpha_{k} \subset \alpha_{l}$ и $a^{*} \alpha_{k} \subset \alpha_{l}$, поэтому $P_{l} P_{k}=P_{k}$ и $P_{l}^{\perp} a^{*} P_{k}=0$. Таким образом,

$$
\left[a^{*}, \sum_{l=1}^{k-1} P_{l}\right] P_{k}=\left(\sum_{l=1}^{k-1} P_{l}^{\perp} a^{*} P_{l}\right) P_{k}=\sum_{l=1}^{k-1} P_{l}^{\perp} a^{*} P_{k}=0 .
$$

Покажем, что справедливы соотношения (3.2). Рассуждая, как выше, получаем, что $\left[a, P_{l}\right]=-P_{l} a P_{l}^{\perp}$ при $l \leqslant n$ и $P_{l}^{\perp} P_{k}=0$ при $l<k$. Отсюда

$$
P_{k}^{\perp}\left(\left[a, \sum_{l=1}^{k-1} P_{l}\right]+a\right) P_{k}=P_{k}^{\perp} a P_{k}=0,
$$

так как $\alpha_{k}-$ BPS-подпространство. 
Мы показали, что $P_{k}$ является унитоном для $\Phi_{k-1}$, следовательно, $\Phi-$ решение и $\Phi=\prod_{j=0}^{n}\left(I-2 P_{j}\right)$ - унитонная факторизация решения $\Phi$ (мы, как и прежде, считаем, что $\left.P_{0}=0\right)$.

Докажем равенства (4.1). Согласно сказанному выше $\Phi(\lambda)=\prod_{j=1}^{n}\left(P_{j}^{\perp}+\lambda P_{j}\right)$ является петлевым поднятием решения $\Phi$. Поскольку $\prod_{j=1}^{n} P_{j}=P_{n} \neq 0$, мы имеем $\operatorname{deg} \Phi(\lambda)=n$, и из теоремы 3.7 работы [5] следует, что $u=n$. Из утверждения 12 работы [3] следует, что $e=\sum_{j=1}^{n} \operatorname{dim} \alpha_{j}$. Покажем, что $r=\sum_{j=1}^{n} r_{j}$. При $n=1$ это равенство очевидно. Пусть $n>1$. По определению $r=\operatorname{dim} \operatorname{im} \Phi_{-}=\operatorname{dim} \operatorname{im} \Phi_{+}$. Так как $\alpha_{j}-$ BPS-подпространство,

$$
\Phi_{+}=2 \sum_{j=1}^{n} P_{j}^{\perp} a^{*} P_{j}
$$

Положим

$$
\frac{1}{2} \Phi_{+}=A+B, \quad A=P_{1}^{\perp} a^{*} P_{1}, \quad B=\sum_{j=2}^{n} P_{j}^{\perp} a^{*} P_{j}
$$

Имеем

$$
A\left(\alpha_{2}\right)=0, \quad B\left(\alpha_{2}^{\perp}\right)=0, \quad \operatorname{im} B \subset \alpha_{1}, \quad \operatorname{im} A \subset \alpha_{1}^{\perp} .
$$

Следовательно,

$$
\operatorname{im}(A+B)=A \alpha_{2}^{\perp} \oplus B \alpha_{2}=\operatorname{im} A \oplus \operatorname{im} B .
$$

Таким образом, $\operatorname{dim} \operatorname{im}(A+B)=\operatorname{dim} \operatorname{im} A+\operatorname{dim} \operatorname{im} B$. Применяя аналогичные соображения, получаем

$$
r=\operatorname{dimim} P_{1}^{\perp} a^{*} P_{1}+\cdots+\operatorname{dimim} P_{n}^{\perp} a^{*} P_{n} .
$$

Так как $r_{j}=\operatorname{dimim}\left(I-2 P_{j}\right)_{+}=\operatorname{dimim} P_{j}^{\perp} a^{*} P_{j}$, мы имеем $r=\sum_{j=1}^{n} r_{j}$. Итак, мы доказали равенства (4.1).

Проверим справедливость неравенств (4.2). Согласно утверждению 2 мы имеем $r_{j} \geqslant r_{j+1}$ и $\operatorname{dim} \alpha_{j} \geqslant \operatorname{dim} \alpha_{j+1}+r_{j}$ для $1 \leqslant j \leqslant n-1$. Учитывая, что $\operatorname{dim} \alpha_{n} \geqslant r_{n}$, последовательно получаем $r_{1} \geqslant r_{2} \geqslant \cdots \geqslant r_{n}$ и $\operatorname{dim} \alpha_{j} \geqslant \sum_{k=j}^{n} r_{k}$. Таким образом,

$$
e=\sum_{j=1}^{n} \operatorname{dim} \alpha_{j} \geqslant \sum_{j=1}^{n} \sum_{k=j}^{n} r_{k}=\sum_{k=1}^{n} k r_{k} \geqslant \sum_{k=1}^{n} r_{k}+\sum_{k=1}^{n}(k-1)=r(\Phi)+\frac{n(n-1)}{2}
$$

что доказывает неравенства (4.2).

ЗАмечание 2 . Ортопроекторы $P_{1}, \ldots, P_{n}$, которые удовлетворяют условиям теоремы 1 , попарно коммутируют, так как $P_{i} P_{j}=P_{j} P_{i}=P_{\max (i, j)}$. Поэтому решение $\Phi=\prod_{j=0}^{n}\left(I-2 P_{j}\right)$ удовлетворяет соотношению $\Phi^{2}=\Phi$. Следовательно, $\Phi$ является отражением, т. е. имеет вид $\Phi=I-2 P$ для некоторого ортопроектора $P$. Такие решения называются грассмановыми.

УтВЕРЖДЕНИЕ 3. Пусть $е, r, n$ - натуральные числа, удовлетворяющие неравенствам

$$
r \geqslant n, \quad e \geqslant r+\frac{n(n-1)}{2} .
$$

Тогда существует решение $\Phi \in M_{0}$ такое, что $е(\Phi)=e, r(\Phi)=r u(\Phi)=n$. 
ДокАЗАТЕЛЬСтво. Если $n=1$, то согласно соотношениям (2.9) всегда существует BPS-решение $\Phi$, для которого $e(\Phi)=e, r(\Phi)=r$, где $e \geqslant r \geqslant 1$.

Пусть $n \geqslant 2$. Положим

$$
r_{1}=r-n+1, \quad k_{1}=e-\frac{n(n-1)}{2}-r_{1}+1
$$

В силу неравенств (4.3) $1 \leqslant r_{1}<r$ и $k_{1} \geqslant n$. Рассмотрим многочлен

$$
\Pi(z)=z^{k_{1}} \prod_{j=1}^{r_{1}-1}(z-j)
$$

(если $r_{1}=1$, то $\left.\Pi(z)=z^{k_{1}}\right)$. В силу формул $(2.8)$ и $(2.9)$ многочлену П( $\left.z\right)$ отвечает BPS-решение $I-2 P_{1}$ с соответствующим подпространством $\alpha_{1}=\operatorname{im} P_{1}=\operatorname{ker} \Pi(a)$. При этом

$$
\alpha_{1} \supset\left\langle e_{0}, \ldots, e_{n-1}\right\rangle, \quad \operatorname{dim} \alpha_{1}=\operatorname{deg} \Pi(z)=e-\frac{n(n-1)}{2}, \quad r\left(I-2 P_{1}\right)=r_{1} .
$$

Возьмем теперь ортопроекторы $P_{j}, 2 \leqslant j \leqslant n$, у которых $\operatorname{im} P_{j}=\alpha_{j}=\left\langle e_{0}, \ldots, e_{n-j}\right\rangle$. По теореме 1 оператор $\Phi=\prod_{j=1}^{n}\left(I-2 P_{j}\right)$ является решением, нормированная энергия и канонический ранг которого равны

$$
e(\Phi)=\sum_{j=1}^{n} \operatorname{dim} \alpha_{j}=e, \quad r(\Phi)=r_{1}+\sum_{j=2}^{n} 1=r,
$$

поэтому построенное $\Phi$ и есть искомое решение.

\section{5. КАНОНИЧЕСКАЯ УНИТОННАЯ ФАКТОРИЗАЦИЯ И ЕЕ СВОЙСТВА}

В этом разделе мы будем рассуждать по аналогии с работами [6], [7].

Поскольку такие целочисленные характеристики решения $\Phi \in M$, как нормированная энергия, канонический ранг и минимальное унитонное число, не меняются при умножении $\Phi$ на константу, равную по модулю единице, мы считаем далее, что $\Phi \in M_{0}$.

Теорема 2. Пусть $\Phi \in M_{0}$ - решение, $n=u(\Phi)$ - его минимальное унитонное число. Тогда существует единственный набор ортопроекторов $P_{0}, P_{1}, \ldots, P_{n}$ maких, что:

а) $\Phi=\prod_{k=0}^{n}\left(I-2 P_{j}\right)$ - унитонная факторизация решения $\Phi$,

б) если $u(\Phi) \neq 0$, то ортопроекторь $P_{0}, P_{1}, \ldots, P_{n}$ удовлетворяют накрьвающим условиям

$$
\operatorname{im} P_{j} P_{j-1} \ldots P_{1}=\operatorname{im} P_{j}, \quad 1 \leqslant j \leqslant n .
$$

ЗАмечаниЕ 3 . Пусть $\alpha_{j}=\operatorname{im} P_{j}, 1 \leqslant j \leqslant n$. Тогда условия (5.1) эквивалентны равенствам

$$
\alpha_{j}^{\perp} \cap \alpha_{j+1}=0, \quad 1 \leqslant j \leqslant n-1 .
$$


ДокАЗАТЕЛЬСтво теоРемы 2. При $n=0$ теорема, очевидно, справедлива. Далее мы считаем, что $n \geqslant 1$.

Докажем существование ортопроекторов $P_{0}, P_{1}, \ldots, P_{n}$. Положим $\Phi_{n}=\Phi, P_{0}=0$. Пусть

$$
\Phi_{n}(\lambda)=\sum_{k=0}^{n} T_{k}^{n} \lambda^{k}
$$

- петлевое поднятие решения $\Phi_{n}, P_{n}$ - ортопроектор такой, что $\operatorname{im} P_{n}=\operatorname{im}\left(T_{n}^{n}\right)^{*}$. Из леммы 3.6 работы [5] следует, что $\operatorname{dim} \operatorname{im} P_{n}<\infty, P_{n}^{\perp}-$ унитон для решения $\Phi_{n}$ и степень петлевого поднятия $\Phi_{n-1}(\lambda)$ решения $\Phi_{n-1}=-\Phi\left(I-2 P_{n}^{\perp}\right)$ равна $n-1$. Имеем $\Phi=\Phi_{n-1}\left(I-2 P_{n}\right)$.

Применяя индукцию, последовательно получаем решения

$$
\Phi_{n-1}, \Phi_{n-2}, \ldots, \Phi_{0} \in M_{0}
$$

с петлевыми поднятиями $\Phi_{j}(\lambda)=\sum_{k=0}^{j} T_{k}^{j} \lambda^{k}$, удовлетворяющими условию $T_{j}^{j} \neq 0$, и набор ортопроекторов $P_{n}, P_{n-1}, \ldots, P_{1}$, удовлетворяющих для всех $j=1, \ldots, n$ соотношениям

$$
\operatorname{im} P_{j}=\operatorname{im}\left(T_{j}^{j}\right)^{*}, \quad \operatorname{dimim} P_{j}<\infty .
$$

При этом ортопроектор $P_{j}$ является унитоном решения $\Phi_{j-1}$ и $\Phi_{j}=\Phi_{j-1}\left(I-2 P_{j}\right)$ для $1 \leqslant j \leqslant n$. Так как $\Phi_{0} \in M_{0}$, справедливо равенство $\Phi_{0}=I=I-2 P_{0}$, следовательно, $\Phi=\prod_{k=0}^{n}\left(I-2 P_{k}\right)$ - унитонная факторизация решения $\Phi$, как сказано в утверждении "a".

Проверим, что построенные ортопроекторы удовлетворяют равенствам (5.1). Поскольку

$$
\Phi_{j}(\lambda)=\prod_{k=0}^{j}\left(P_{k}^{\perp}+\lambda P_{k}\right)=\sum_{k=0}^{j} T_{k}^{j} \lambda^{k}
$$

мы имеем $T_{j}^{j}=\prod_{k=1}^{j} P_{k}$ при $1 \leqslant j \leqslant n$. Применив соотношения (5.3), получаем

$$
\operatorname{im} P_{j}=\operatorname{im}\left(T_{j}^{j}\right)^{*}=\operatorname{im} \prod_{k=0}^{j-1} P_{j-k},
$$

то есть имеет место формула (5.1).

Покажем, что набор ортопроекторов, удовлетворяющих условиям "а" и "б" теоремы, единствен. Пусть $\pi_{0}, \pi_{1}, \ldots, \pi_{n}$ - некоторый набор ортопроекторов, удовлетворяющих условиям "а" и "б". Покажем, что $\pi_{j}=P_{j}$ для всех $j=0,1, \ldots, n$. Очевидно, $\pi_{0}=P_{0}=0$. Согласно условию "a" $\Phi(\lambda)=\prod_{k=0}^{n}\left(\pi_{k}^{\perp}+\lambda \pi_{k}\right)$, поэтому $T_{n}^{n}=\prod_{k=1}^{n} \pi_{k}$. В силу (5.3) ортопроектор $P_{n}$ удовлетворяет соотношениям

$$
\operatorname{im} P_{n}=\operatorname{im}\left(T_{n}^{n}\right)^{*}=\operatorname{im} \prod_{k=0}^{n-1} \pi_{n-k}=\operatorname{im} \pi_{n}
$$

(последнее равенство справедливо, поскольку ортопроекторы $\pi_{0}, \pi_{1}, \ldots, \pi_{n}$ удовлетворяют условию "б", а значит, и условиям (5.1)). Таким образом, мы показали, что $P_{n}=\pi_{n}$. 
Применяя индукцию, последовательно получаем

$$
\begin{array}{rlrl}
\Phi_{n-1}(\lambda) & =\prod_{k=0}^{n-1}\left(\pi_{k}^{\perp}+\lambda \pi_{k}\right), & & \pi_{n-1}=P_{n-1}, \\
\ldots \ldots \ldots \ldots \ldots \ldots \ldots, & & \\
\Phi_{1}(\lambda) & =\pi_{1}^{\perp}+\lambda \pi_{1}, & \pi_{1}=P_{1} .
\end{array}
$$

Теорема доказана.

ОПРеДЕЛЕНИЕ 1 . Пусть $\Phi \in M_{0}$ и $P_{0}, P_{1}, \ldots, P_{n}$ - набор унитонов, удовлетворяющих условиям теоремы 2 . Тогда представление $\Phi=\prod_{j=0}^{n}\left(I-2 P_{j}\right)$ назовем канонической унитонной факторизацией решения $\Phi$.

Теорема 3. Пусть $\Phi=\prod_{j=0}^{n}\left(I-2 P_{j}\right)$ - каноническая унитонная факторизаиия решения $\Phi \in M_{0}$. Тогда для всех $j=1, \ldots, n$ справедливы равенства

$$
\begin{gathered}
P_{j}\left[a, \sum_{k=0}^{j-1} P_{k}\right]=0 \\
{\left[a, \sum_{k=1}^{j} P_{k}\right]=\left[a, \sum_{k=1}^{j} P_{k}\right] P_{j}^{\perp} .}
\end{gathered}
$$

ЗАмечание 4. Условие (5.4) эквивалентно $P_{j}\left(\Phi_{j-1}\right)_{-}=0$, где, как и раньше, $\Phi_{j-1}=\prod_{k=0}^{j-1}\left(I-2 P_{k}\right)$. Поэтому унитон $P_{j}$ является базовым для $\Phi_{j-1}$ (см. работу [3], определение 6).

ДоказАТЕЛЬСТво теоремы 3 . Положим, как раньше, для $1 \leqslant j \leqslant n$

$$
\alpha_{j}=\operatorname{im} P_{j}, \quad \Phi_{j-1}=\prod_{k=0}^{j-1}\left(I-2 P_{k}\right) .
$$

Применим индукцию по $j$. При $j=1$ равенство (5.4) справедливо, ибо $P_{0}=0$. Так как $P_{1}$ является унитоном для $\Phi_{0}=I$, согласно формуле $(2.3) P_{1}^{\perp} a P_{1}=0$, следовательно, $\left[a, P_{1}\right]=-P_{1} a P_{1}^{\perp}=\left[a, P_{1}\right] P_{1}^{\perp}$, т. е. равенство (5.5) также справедливо.

Предположим, что равенства (5.4) и (5.5) выполнены при $j \leqslant l<n$. Покажем, что эти соотношения справедливы при $j=l+1$.

Ортопроектор $P_{l+1}-$ унитон для $\Phi_{l}$ и при $j=l$ справедливо $(5.5)$, поэтому равенство (3.1) влечет

$$
0=P_{l+1}\left[a, \sum_{k=1}^{l} P_{k}\right] P_{l+1}^{\perp}=P_{l+1}\left[a, \sum_{k=1}^{l} P_{k}\right] P_{l}^{\perp} P_{l+1}^{\perp},
$$

что эквивалентно

$$
P_{l+1}^{\perp} P_{l}^{\perp}\left(\left[a, \sum_{k=1}^{l} P_{k}\right]\right)^{*} P_{l+1}=0 .
$$

Учитывая соотношение (5.2), получаем

$$
\operatorname{im} P_{l}^{\perp}\left(\left[a, \sum_{k=1}^{l} P_{k}\right]\right)^{*} P_{l+1} \subset \alpha_{l+1} \cap \alpha_{l}^{\perp}=0 .
$$


Переходя к сопряженному равенству, имеем $P_{l+1}\left[a, \sum_{k=1}^{l} P_{k}\right] P_{l}^{\perp}=0$. Вновь применив (5.5) при $j=l$, получаем $P_{l+1}\left[a, \sum_{k=1}^{l} P_{k}\right]=0$, следовательно, равенство (5.4) справедливо при $j=l+1$.

Докажем соотношение (5.5) при $j=l+1$. В силу (3.2)

$$
P_{l+1}^{\perp}\left[a, \sum_{k=1}^{l} P_{k}\right] P_{l+1}=-P_{l+1}^{\perp} a P_{l+1} .
$$

Равенство (5.4) при $j=l+1$ эквивалентно

$$
\left[a, \sum_{k=1}^{l} P_{k}\right]=P_{l+1}^{\perp}\left[a, \sum_{k=1}^{l} P_{k}\right] .
$$

Используя формулы (5.6) и (5.7), получаем

$$
\begin{gathered}
{\left[a, \sum_{k=1}^{l} P_{k}\right]=P_{l+1}^{\perp}\left[a, \sum_{k=1}^{l} P_{k}\right]=P_{l+1}^{\perp}\left[a, \sum_{k=1}^{l} P_{k}\right] P_{l+1}+P_{l+1}^{\perp}\left[a, \sum_{k=1}^{l} P_{k}\right] P_{l+1}^{\perp}=} \\
=-P_{l+1}^{\perp} a P_{l+1}+P_{l+1}^{\perp}\left[a, \sum_{k=1}^{l} P_{k}\right] P_{l+1}^{\perp}=-P_{l+1}^{\perp} a P_{l+1}+\left[a, \sum_{k=1}^{l} P_{k}\right] P_{l+1}^{\perp} .
\end{gathered}
$$

Добавляя $\left[a, P_{l+1}\right]$ к левой и правой частям равенства (5.8) и учитывая, что

$$
-P_{l+1}^{\perp} a P_{l+1}=-\left[a, P_{l+1}\right] P_{l+1},
$$

окончательно получаем

$$
\left[a, \sum_{k=1}^{l+1} P_{k}\right]=\left[a, \sum_{k=1}^{l} P_{k}\right] P_{l+1}^{\perp}-\left[a, P_{l+1}\right] P_{l+1}+\left[a, P_{l+1}\right]=\left[a, \sum_{k=1}^{l+1} P_{k}\right] P_{l+1}^{\perp},
$$

что и требовалось доказать.

Лемма 1. Пусть $\Phi=\prod_{j=0}^{n}\left(I-2 P_{j}\right)-$ каноническая унитонная факторизация решения $\Phi \in M_{0}$ и $n \geqslant 2$. Тогда

$$
P_{j-2} P_{j-1} a^{*} P_{j}=P_{j-2} a^{*} P_{j-1} P_{j}, \quad 2 \leqslant j \leqslant n .
$$

ДоказАтЕЛЬСтво. При $j=2$ утверждение очевидно. Пусть $j>2$. Имеем

$$
P_{j-1} a^{*} P_{j}=a^{*} P_{j-1} P_{j}-\left[a^{*}, P_{j-1}\right] P_{j}=a^{*} P_{j-1} P_{j}-\left[a^{*}, \sum_{k=0}^{j-1} P_{k}\right] P_{j}+\left[a^{*}, \sum_{k=0}^{j-2} P_{k}\right] P_{j} .
$$

Учитывая равенства (5.4) и (5.5), получаем

$$
P_{j-1} a^{*} P_{j}=a^{*} P_{j-1} P_{j}+P_{j-2}^{\perp}\left[a^{*}, \sum_{k=0}^{j-2} P_{k}\right] P_{j} .
$$

Остается применить к обеим частям этого равенства ортопроектор $P_{j-2}$, и лемма доказана. 
ТеОрема 4. Пусть $\Phi=\prod_{j=0}^{n}\left(I-2 P_{j}\right)$ - каноническая унитонная бакторизация решения $\Phi \in M_{0} u n \geqslant 1$. Положим $\beta_{j}=\operatorname{im} P_{1} \ldots P_{j}, 1 \leqslant j \leqslant n$. Тогда имеют место следующие утверждения:

а) $a^{*} \beta_{j} \subset \beta_{j-1}$ для всех $j=2, \ldots, n$,

б) $\beta_{j}-$ BPS-подпространство для всех $j=1, \ldots, n$.

ДоказАтельство. Докажем утверждение "а". Мы имеем $\left[a^{*}, P_{1}\right] P_{2}=0$ в силу равенства (5.4), отсюда

$$
a^{*} P_{1} P_{2}=\left[a^{*}, P_{1}\right] P_{2}+P_{1} a^{*} P_{2}=P_{1} a^{*} P_{2},
$$

поэтому $a^{*} \beta_{2} \subset \beta_{1}$. Пусть теперь $j \geqslant 3$. Применив соотношение (5.9), последовательно получаем $P_{1} a^{*} P_{2} P_{3}=P_{1} P_{2} a^{*} P_{3}, P_{2} a^{*} P_{3} P_{4}=P_{2} P_{3} a^{*} P_{4}$ и т. д. В силу (5.11)

$$
a^{*} \prod_{l=1}^{j} P_{l}=P_{1} a^{*} \prod_{l=2}^{j} P_{l}=P_{1} P_{2} a^{*} \prod_{l=3}^{j} P_{l}=\cdots=\left(\prod_{l=1}^{j-1} P_{l}\right) a^{*} P_{j} .
$$

Отсюда $a^{*} \beta_{j} \subset \beta_{j-1}$.

Докажем утверждение "б". Положим, как и прежде, $\alpha_{j}=\operatorname{im} P_{j}$. Если $j=1$, то согласно (3.2) $\beta_{1}=\alpha_{1}$ - BPS-подпространство. Пусть $j>1$. Покажем по индукции, что для $1 \leqslant k \leqslant j-1$

$$
P_{j-k} a P_{j-k} P_{j-k+1} \ldots P_{j-1} \alpha_{j} \subset P_{j-k} P_{j-k+1} \ldots P_{j-1} \alpha_{j} .
$$

Проверим сначала справедливость условия (5.13) при $k=1$. Из равенства (5.4) следует, что

$$
P_{l}\left[a, \sum_{q=1}^{l} P_{q}\right]=P_{l}\left[a, P_{l}\right], \quad 1 \leqslant l \leqslant n .
$$

Запишем условие (3.2) в виде

$$
\left(a+\left[a, \sum_{q=1}^{j-1} P_{q}\right]\right) \alpha_{j} \subset \alpha_{j}
$$

Применяя ортопроектор $P_{j-1}$ к обеим частям этого соотношения и учитывая равенство (5.14) при $l=j-1$, получаем

$$
P_{j-1} a P_{j-1} \alpha_{j} \subset P_{j-1} \alpha_{j},
$$

другими словами, включение (5.13) выполнено при $k=1$.

Допустим, что включение (5.13) справедливо при $1 \leqslant k \leqslant k_{0}<j-1$. Проверим, что оно имеет место и при $k=k_{0}+1$. В силу (3.2) и (5.4)

$$
\left(\left[a, \sum_{q=1}^{j-k_{0}-1} P_{q}\right]+P_{j-k_{0}}^{\perp} a\right) P_{j-k_{0}}=0,
$$

в частности

$$
\left(\left[a, \sum_{q=1}^{j-k_{0}-1} P_{q}\right]+P_{j-k_{0}}^{\perp} a\right) P_{j-k_{0}} P_{j-k_{0}+1} \ldots P_{j-1} \alpha_{j}=0 .
$$


Добавим к этому равенству включение (5.13), взятое при $k=k_{0}$ : имеем

$$
\left(\left[a, \sum_{q=1}^{j-k_{0}-1} P_{q}\right]+a\right) P_{j-k_{0}} P_{j-k_{0}+1} \ldots P_{j-1} \alpha_{j} \subset P_{j-k_{0}} P_{j-k_{0}+1} \ldots P_{j-1} \alpha_{j} .
$$

Применяя к обеим частям данного соотношения ортопроектор $P_{j-k_{0}-1}$ и используя равенство (5.14), взятое при $l=j-k_{0}-1$, получаем искомое включение (5.13) при $k=k_{0}+1$.

Итак, мы показали, что включение (5.13) справедливо при всех $k=1, \ldots, j-1$. При $k=j-1$ оно имеет вид

$$
P_{1} a P_{1} \ldots P_{j-1} \alpha_{j} \subset P_{1} \ldots P_{j-1} \alpha_{j}
$$

$\mathrm{B}$ соответствии с формулой (3.2) $P_{1} a P_{1}=a P_{1}$, поэтому $a \beta_{j} \subset \beta_{j}$, т. е. $\beta_{j}$ является BPS-подпространством. Теорема доказана.

ОПреДЕЛЕНИЕ 2. Пусть $\Phi \in M_{0}$ - решение, $n=u(\Phi) \geqslant 1$, подпространства $\beta_{1}, \ldots, \beta_{n}$ определены, как в теореме 4, и $\pi_{1}, \ldots, \pi_{n}$ - отвечающие им ортопроекторы (т. е. $\operatorname{im} \pi_{j}=\beta_{j}$ для $\left.1 \leqslant j \leqslant n\right)$. Оператор $\widetilde{\Phi}=\prod_{j=1}^{n}\left(I-2 \pi_{j}\right)$ назовем плоским решением, отвечающим решению $\Phi^{2)}$.

Теорема 5. Пусть $\Phi \in M_{0}$ - решение, $u(\Phi) \geqslant 1, \widetilde{\Phi}-$ плоское решение, отвечающее $\Phi$. Тогда

$$
u(\widetilde{\Phi})=u(\Phi), \quad e(\widetilde{\Phi})=e(\Phi), \quad r(\widetilde{\Phi}) \leqslant r(\Phi)
$$

Доказательство. Пусть $n=u(\Phi), \Phi=\prod_{k=0}^{n}\left(I-2 P_{k}\right)-$ каноническая унитонная факторизация решения $\Phi, \alpha_{j}=\operatorname{im} P_{j}, \widetilde{\Phi}=\prod_{j=1}^{n}\left(I-2 \pi_{j}\right)$ - плоское решение, отвечающее $\Phi$. Так как $P_{j} \neq 0$, в силу накрывающих условий (5.1) $\beta_{j} \neq 0$, следовательно, $u(\widetilde{\Phi})=n$ по теореме 1 .

Так как

$$
e(\Phi)=\sum_{j=1}^{n} \operatorname{dim} \alpha_{j}, \quad e(\widetilde{\Phi})=\sum_{j=1}^{n} \operatorname{dim} \beta_{j},
$$

равенство $e(\widetilde{\Phi})=e(\Phi)$ будет следовать из того, что

$$
\operatorname{dim} \alpha_{j}=\operatorname{dim} \beta_{j}, \quad 1 \leqslant j \leqslant n .
$$

Имеем $\alpha_{1}=\beta_{1}$ и $\beta_{j}=P_{1} \ldots P_{j-1} \alpha_{j}$ при $j>1$. Поэтому равенство (5.15) верно при $j=1$, а для $j>1$ нам достаточно показать, что оператор $P_{1} \ldots P_{j-1}$ является взаимно однозначным на $\alpha_{j}$. Допустим, что $P_{1} \ldots P_{j-1} x=0$ для некоторого $x \in \alpha_{j}$. Тогда в силу соотношений (5.2) при $j=2$ имеем $x \in \alpha_{2} \cap \alpha_{1}^{\perp}=0$, а при $j>2$ имеем $P_{2} \ldots P_{j-1} x \in \alpha_{2} \cap \alpha_{1}^{\perp}=0$. С помощью аналогичных рассуждений получаем, что $x \in \alpha_{3} \cap \alpha_{2}^{\perp}=0$ при $j=3$ и $P_{3} \ldots P_{j-1} x \in \alpha_{3} \cap \alpha_{2}^{\perp}=0$ и т. д. В результате получаем, что $x \in \alpha_{j} \cap \alpha_{j-1}^{\perp}=0$.

Докажем, что $r(\widetilde{\Phi}) \leqslant r(\Phi)$. Для каждого $j=1, \ldots, n$ положим, как и раньше, $\Phi_{j}=\prod_{k=1}^{j}\left(I-2 P_{k}\right)$. Тогда $\widetilde{\Phi}_{j}=\prod_{k=1}^{j}\left(I-2 \pi_{k}\right)$ - плоское решение, отвечающее $\Phi_{j}$.

\footnotetext{
2) Оператор $\widetilde{\Phi}$ является решением по теореме 1 .
} 
Покажем, что $r\left(\Phi_{j}\right) \geqslant r\left(\widetilde{\Phi}_{j}\right)$ для всех $j=1, \ldots, n$. Так как $\alpha_{1}=\beta_{1}$, то $\Phi_{1}=\widetilde{\Phi}_{1}$, тем самым $r\left(\Phi_{1}\right)=r\left(\widetilde{\Phi}_{1}\right)$. Предположим, что $r\left(\widetilde{\Phi}_{j}\right) \leqslant r\left(\Phi_{j}\right)$ при $1 \leqslant j<l$. Покажем, что $r\left(\widetilde{\Phi}_{l}\right) \leqslant r\left(\Phi_{l}\right)$.

Пусть $r_{l}=r\left(I-2 \pi_{l}\right)$. Поскольку $\beta_{l}-$ BPS-подпространство, в силу (2.8) мы имеем

$$
\beta_{l}=\left\langle R_{\lambda_{j}}^{q_{j}-1} \mid 1 \leqslant j \leqslant r_{l}, 1 \leqslant q_{j} \leqslant k_{j}\right\rangle
$$

для некоторых попарно различных комплексных чисел $\lambda_{1}, \ldots, \lambda_{r_{l}}$ и некоторых натуральных $k_{1}, \ldots, k_{r_{l}}$.

В силу доказанного выше оператор $P_{1} \ldots P_{l-1}: \alpha_{l} \rightarrow \beta_{l}$ обратим, и поэтому при $1 \leqslant j \leqslant r_{l}$ существует единственный вектор $x_{j} \in \alpha_{l}$ такой, что $P_{1} \ldots P_{l-1} x_{j}=R_{\lambda_{j}}^{k_{j}-1}$. Векторы $x_{1}, \ldots, x_{r_{l}}$ линейно независимы. Рассмотрим подпространство

$$
\mathcal{L}=\alpha_{l}^{\perp}+\left\langle x_{1}, \ldots, x_{r_{l}}\right\rangle .
$$

Согласно соотношению $(2.5) r\left(\Phi_{l}\right)=\operatorname{dim} \operatorname{im} \Phi_{l+} \geqslant \operatorname{dim} \Phi_{l_{+}} \mathcal{L}$. В силу $(5.5)$

$$
\frac{1}{2} \Phi_{l+}=\left[a^{*}, \sum_{j=1}^{l} P_{j}\right]=P_{l}^{\perp}\left[a^{*}, \sum_{j=1}^{l} P_{j}\right]=P_{l}^{\perp}\left(\left[a^{*}, \sum_{j=1}^{l-1} P_{j}\right]+a^{*} P_{l}\right) .
$$

Сначала докажем включение $\operatorname{ker} \Phi_{l+} \cap \mathcal{L} \subset \alpha_{l}^{\perp}$, из которого следует неравенство $r\left(\Phi_{l}\right) \geqslant \operatorname{dim} \Phi_{l+} \alpha_{l}^{\perp}+r_{l}$. Возьмем $x \in \operatorname{ker} \Phi_{l+} \cap \mathcal{L}$. Из соотношения (5.16) имеем

$$
\left[a^{*}, \sum_{j=1}^{l-1} P_{j}\right] x+a^{*} P_{l} x \in \alpha_{l}
$$

Согласно равенству (5.5) $\left[a^{*}, \sum_{j=1}^{l-1} P_{j}\right] x \in \alpha_{l-1}^{\perp}$, поэтому, применив к обеим частям (5.17) суперпозицию $P_{1} \ldots P_{l-1}$, получим

$$
P_{1} \ldots P_{l-1} a^{*} P_{l} x \subset P_{1} \ldots P_{l-1} \alpha_{l}=\beta_{l} .
$$

Учитывая формулу (5.12), заключаем, что $a^{*} P_{1} \ldots P_{l} x \in \beta_{l}$.

Так как $x \in \mathcal{L}$,

$$
P_{l} x \in\left\langle x_{1}, \ldots, x_{r_{l}}\right\rangle, \quad P_{1} \ldots P_{l} x \in\left\langle R_{\lambda_{j}}^{k_{j}-1} \mid 1 \leqslant j \leqslant r_{l}\right\rangle,
$$

в частности, $P_{1} \ldots P_{l} x=0$ тогда и только тогда, когда $P_{l} x=0$. Имеем

$$
a^{*} \prod_{j=1}^{l} P_{j} x \in\left\langle a^{*} \prod_{j=1}^{l} P_{j} x_{q} \mid 1 \leqslant q \leqslant r_{l}\right\rangle .
$$

Из соотношений

$$
a^{*} \prod_{i=1}^{l} P_{i} x_{q}=a^{*} R_{\lambda_{q}}^{k_{q}-1}=R_{\lambda_{q}}^{k_{q}}+\bar{\lambda}_{q} R_{\lambda_{q}}^{k_{q}-1}, \quad a^{*} \prod_{i=1}^{l} P_{i} x \in \beta_{l}
$$

вытекает $a^{*} \prod_{i=1}^{l} P_{i} x=0$, следовательно, $\prod_{i=1}^{l} P_{i} x=0$ и $P_{l} x=0$, т. е. $x \in \alpha_{l}^{\perp}$. 
Далее, покажем, что $\operatorname{dim} \Phi_{l_{+}} \alpha_{l}^{\perp}=r\left(\Phi_{l-1}\right)$. Тогда по предположению индукции и с учетом доказанного выше получим, что

$$
r\left(\Phi_{l}\right) \geqslant r_{l}+r\left(\Phi_{l-1}\right) \geqslant r_{l}+r\left(\widetilde{\Phi}_{l-1}\right)=r\left(\widetilde{\Phi}_{l}\right)
$$

(последнее равенство следует из теоремы 1).

Согласно (5.4)

$$
\left[a^{*}, \sum_{j=1}^{l-1} P_{j}\right] \alpha_{l}^{\perp}=\operatorname{im}\left[a^{*}, \sum_{j=1}^{l-1} P_{j}\right] .
$$

Применяя формулу (5.16), получаем

$$
\frac{1}{2} \Phi_{l+} \alpha_{l}^{\perp}=P_{l}^{\perp}\left[a^{*}, \sum_{j=1}^{l-1} P_{j}\right] \alpha_{l}^{\perp}=\operatorname{im} P_{l}^{\perp}\left[a^{*}, \sum_{j=1}^{l-1} P_{j}\right] .
$$

Поскольку

$$
r\left(\Phi_{l-1}\right)=\operatorname{dimim}\left(\Phi_{l-1}\right)_{+}=\operatorname{dimim}\left[a^{*}, \sum_{j=1}^{l-1} P_{j}\right] \geqslant \operatorname{dimim} P_{l}^{\perp}\left[a^{*}, \sum_{j=1}^{l-1} P_{j}\right],
$$

достаточно показать, что

$$
\operatorname{ker} P_{l}^{\perp}\left[a^{*}, \sum_{j=1}^{l-1} P_{j}\right]=\operatorname{ker}\left[a^{*}, \sum_{j=1}^{l-1} P_{j}\right] .
$$

Последнее вытекает из условий (5.2):

$$
P_{l}^{\perp}\left[a^{*}, \sum_{j=1}^{l-1} P_{j}\right] z=0 \Longrightarrow\left[a^{*}, \sum_{j=1}^{l-1} P_{j}\right] z \in \alpha_{l} .
$$

Но $\operatorname{im}\left[a^{*}, \sum_{j=1}^{l-1} P_{j}\right] \subset \alpha_{l-1}^{\perp}$, следовательно, $\left[a^{*}, \sum_{j=1}^{l-1} P_{j}\right] z \in \alpha_{l} \cap \alpha_{l-1}^{\perp}=0$. Теорема доказана.

СлеДСТвИЕ 1. Пусть $\Phi \in M_{0}$ - решение. Тогда

$$
r(\Phi) \geqslant u(\Phi), \quad e(\Phi) \geqslant \frac{u(\Phi)(u(\Phi)+1)}{2} .
$$

ДОКАЗАТЕЛЬСТво. Если $u(\Phi)=0$, то $\Phi=I$ и $r(\Phi)=e(\Phi)=0$. Теперь пусть $n=u(\Phi) \geqslant 1$. Рассмотрим плоское решение $\widetilde{\Phi}$, отвечающее $\Phi$. По теореме 5 мы имеем $r(\Phi) \geqslant r(\widetilde{\Phi}), e(\Phi)=e(\widetilde{\Phi}), u(\Phi)=u(\widetilde{\Phi})=n$.

Решение $\widetilde{\Phi}$ удовлетворяет условиям теоремы 1 , отсюда получаем неравенства

$$
r(\widetilde{\Phi}) \geqslant n, \quad e(\widetilde{\Phi}) \geqslant \frac{n(n-1)}{2}+r(\widetilde{\Phi}) \geqslant \frac{n(n-1)}{2}+n=\frac{n(n+1)}{2} .
$$

Следовательно, $r(\Phi) \geqslant n$ и $е(\Phi) \geqslant n(n+1) / 2$.

Благодарности. Работа выполнена при финансовой поддержке РФФИ (грант № 11-01-00495_а). 


\section{Список литературы}

[1] O. Lechtenfeld, A. D. Popov, JHEP, 11 (2001), 40, 32 pp., arXiv: hep-th/0106213.

[2] A.V. Domrin, O. Lechtenfeld, S. Petersen, JHEP, 03 (2005), 045, 34 pp., arXiv: hep-th/0412001.

[3] А. В. Домрин, ТМФ, 154:2 (2008), 220-239.

[4] А. В. Домрин, ТМФ, 156:3 (2008), 307-327.

[5] А. В. Домрина, Тр. МИАН, 279 (2012), 72-80.

[6] M. J. Ferreira, B. A. Simões, J. C. Wood, Math. Z., 266:4 (2010), 953-978.

[7] K. Uhlenbeck, J. Differential Geom., 30:1 (1989), 1-50.

Поступила в редакцию 10.09.2013 